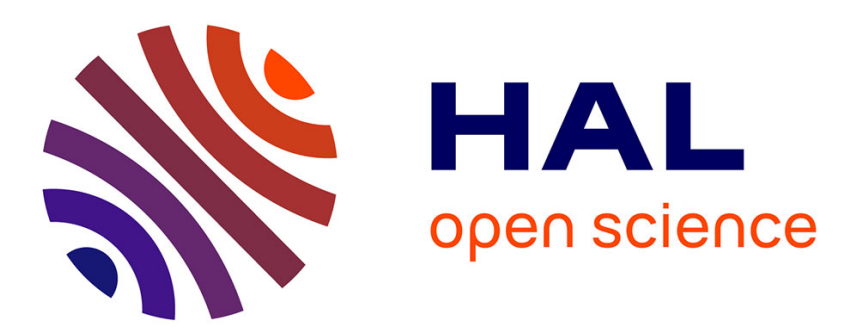

\title{
Heterogeneity and the Role of Normal Stresses during the Extensional Thinning of Non-Brownian Shear-Thickening Fluids
}

\author{
Matthieu Roché, Hamid Kellay, Howard A. Stone
}

\section{- To cite this version:}

Matthieu Roché, Hamid Kellay, Howard A. Stone. Heterogeneity and the Role of Normal Stresses during the Extensional Thinning of Non-Brownian Shear-Thickening Fluids. Physical Review Letters, 2011, 107 (13), pp.134503. 10.1103/PhysRevLett.107.134503 . hal-00668417

\section{HAL Id: hal-00668417 https://hal.science/hal-00668417}

Submitted on 15 Dec 2017

HAL is a multi-disciplinary open access archive for the deposit and dissemination of scientific research documents, whether they are published or not. The documents may come from teaching and research institutions in France or abroad, or from public or private research centers.
L'archive ouverte pluridisciplinaire HAL, est destinée au dépôt et à la diffusion de documents scientifiques de niveau recherche, publiés ou non, émanant des établissements d'enseignement et de recherche français ou étrangers, des laboratoires publics ou privés. 


\title{
Heterogeneity and the Role of Normal Stresses during the Extensional Thinning of Non-Brownian Shear-Thickening Fluids
}

\author{
Matthieu Roché, ${ }^{1}$ Hamid Kellay, ${ }^{2}$ and Howard A. Stone ${ }^{1, *}$ \\ ${ }^{1}$ Department of Mechanical and Aerospace Engineering, Princeton University, Princeton, New Jersey 08544, USA \\ ${ }^{2}$ Université Bordeaux 1, Laboratoire Ondes et Matière d'Aquitaine, CNRS UMR 5798, \\ 351 cours de la Libération, 33405, Talence, France
}

(Received 13 May 2011; published 23 September 2011)

\begin{abstract}
We contrast the extensional and shear dynamics of non-Brownian suspensions as a function of particle concentration. We show that the thinning rate selected during the viscoelastic pinch-off of a liquid bridge is related to the shear rate at which normal stresses become positive, which differs from the shear rate at the onset of shear thickening. By tracking particles, we demonstrate that the extensional flow is heterogeneous, with local variations of the volume fraction consistent with self-dilution. This nonuniform structure is the cause of the buckling of the threads formed after breakup.
\end{abstract}

PACS numbers: 47.50.Gj, 47.57.E-, 47.57.Qk, 83.80.Hj

One measure of the resistance of a fluid to flow is viscosity. This property is usually measured in shear or extension. In shear, two plates separated by a gap $w$ move parallel to each other at speed $V$, which results in a shear rate $\dot{\gamma}=V / w$, while in extension, two plates separated by a gap $L$ are pulled apart at an extension rate $\dot{\epsilon}=$ $L^{-1} d L / d t$. The former provides the shear viscosity $\eta_{s}$ while the latter yields the extensional viscosity $\eta_{e}$. For common Newtonian fluids, $\eta_{s}$ and $\eta_{e}$ are independent of strain rate and are related by $\eta_{e}=3 \eta_{s}$. The picture is more complex for non-Newtonian fluids. For polymer solutions, $\eta_{s}$ can decrease with increasing $\dot{\gamma}$, whereas $\eta_{e}$ increases with increasing $\dot{\epsilon}[1]$. Another example is provided by concentrated particle suspensions for which both $\eta_{s}$ and $\eta_{e}$ increase dramatically when the strain rate exceeds a critical value $\dot{\gamma}_{c}$ in shear (shear thickening) [2,3] and $\dot{\epsilon}_{c}$ in extension (strain hardening) [4-6]. This viscosity increase is widely studied as it can impair the industrial processing of suspensions $[2,3]$.

Shear thickening is well understood for both Brownian [7-9] and non-Brownian suspensions [10-13]. The observation of shear thickening depends on the magnitude of the yield stress $\sigma_{c}$, which in turn depends on the interactions between the particles and the volume fraction $\phi[11,12]$. The onset of shear thickening is associated with the growth of particle clusters for Brownian suspensions [9] and with particle migration towards regions of lower shear rates for non-Brownian suspensions $[10,13]$. Shear thickening is often accompanied by an increase in normal stresses $[2,10]$. The suspensions jam eventually. The magnitude of $\dot{\gamma}_{c}$ decreases when the suspensions are more and more confined [10]. Therefore, understanding the shear response of suspensions requires accounting for flow-induced heterogeneities in the particle volume fraction and the degree of confinement of the particles.

During extensional flow, concentrated suspensions form a cylindrical bridge, which thins exponentially with time
[4-6]. At the highest achievable extension rates, the bridge can fracture $[4,6]$, which supports the occurrence of jamming. The extensional response is believed to rely on the same microscopic mechanism as that in shear, but direct evidence is missing.

In this Letter, we clarify the relation between shear flow and extensional flow of suspensions by studying the pinchoff of non-Brownian suspensions. We characterize the thinning dynamics of a liquid bridge and measure as a function of time $t$ the extension rate $\dot{\epsilon}(t)$ and the strain $\epsilon(t)$. For the most concentrated suspensions, we show that the relaxation rate, extracted from the thinning dynamics, compares well with the shear rate $\dot{\gamma}_{N}$ at which a positive normal stress difference is measured. Close to breakup, we observe fluctuations on the interface of the bridge. Using high-speed microscopy, we track the particles and demonstrate that these fluctuations are related to heterogeneities in the flow. Certain regions of the bridge become jammed while particles experience a significant flow in other regions. We provide evidence that this response is a consequence of a nonuniform particle volume fraction along the axis of the bridge, which we interpret as the result of selfdilution [14]. The heterogeneous structure allows us to explain why the threads observed after breakup buckle while they retract.

We performed experiments with aqueous suspensions of corn starch (Sigma Aldrich). We added cesium chloride $(\mathrm{CsCl},[\mathrm{CsCl}]=55 \mathrm{wt} \%)$ to match the density of the particles, $\rho \simeq 1590 \mathrm{~kg} \mathrm{~m}^{-3}$ [15]. The particles are irregular in shape and have an average diameter $d_{p}=14 \mu \mathrm{m}$. The particle concentration was varied in the range 23 wt $\% \leq[C S] \leq 39$ wt \%; suspensions with higher concentrations flowed nonsteadily. Because of density matching, $[C S]$ is also a measure of the particle volume fraction $\phi$. The shear viscosity $\eta_{s}$ of the suspensions was measured using a plate-plate geometry (diameter $d=50 \mathrm{~mm}$, variable gap width $w$ ) on a rheometer (MCR 301, Anton Paar) 
at temperature $T=25 \pm 0.1^{\circ} \mathrm{C}$ with attention paid to avoid surplus effects [10]. The suspensions are Newtonian for $[C S]<27$ wt $\%$ and non-Newtonian for $[C S] \geq 27$ wt \%, with a shear-thinning regime at low shear rates followed by continuous shear thickening
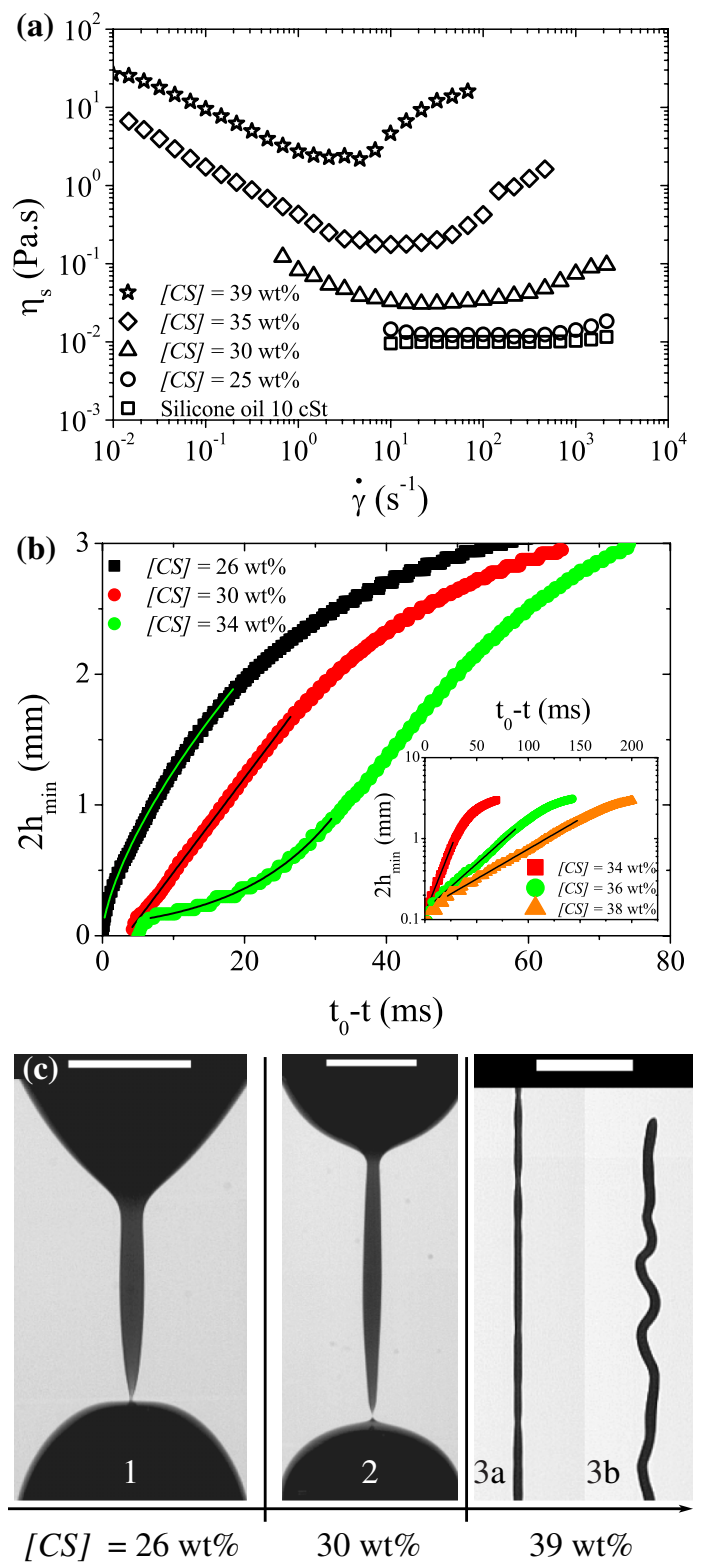

FIG. 1 (color online). (a) Shear rheology of the suspensions, measured with a gap width $w=1 \mathrm{~mm}$. The viscosity of a 10-cSt silicone oil is shown as a calibration for the measurements of low viscosities. (b) Thinning dynamics $2 h_{\min }$ versus $\left(t_{0}-t\right)$ for three different suspensions, where $t_{0}$ is the pinch-off time. For clarity, time is shifted by $4 \mathrm{~ms}$ for $[C S]=30 \mathrm{wt} \%$ and by $5 \mathrm{~ms}$ for $[C S]=34 \mathrm{wt} \%$. Continuous lines: fits to the experimental data; $h_{\min } \propto\left(t_{0}-t\right)^{2 / 3}$ for $[C S]=26 \mathrm{wt} \%, h_{\min } \propto\left(t_{0}-t\right)$ for $[C S]=30 \mathrm{wt} \%$ and $h_{\min } \propto \exp \left(\alpha\left(t_{0}-t\right)\right)$ for $[C S]=34 \mathrm{wt} \%$. Inset: semilog plot of $h_{\min }$ versus $\left(t_{0}-t\right)$ in the viscoelastic regime. (c) Pinch-off of the suspensions. Image 1: inviscidlike; image 2: viscouslike; image 3: viscoelasticlike. Scale bar: $2 \mathrm{~mm}$.
[Fig. 1(a)]. These suspensions have an estimated yield stress $\sigma_{c} \leq 0.5 \mathrm{~Pa}$ [see Fig. (a) in Supplemental Material [16]]. Using the pendant drop method, we measured the surface tension for each of the suspensions $\gamma=$ $(73.9 \pm 1.3) \times 10^{-3} \mathrm{~N} \mathrm{~m}^{-1}$, which is equal to the surface tension of the $\mathrm{CsCl}$ solution and higher than the surface tension of ultrapure water measured with the same setup, $\gamma_{w}=(69.4 \pm 1.4) \times 10^{-3} \mathrm{~N} \mathrm{~m}^{-1}$, because of the added salt. The particles did not have any detectable surface effect.

A syringe pump supplied the suspensions at the needle tip (inner radius $r_{\text {in }}=1.9 \mathrm{~mm}$ ) at a flow rate $Q=$ $0.1 \mathrm{~mL} \mathrm{~min}^{-1}$. Close to the tip, we estimate the Reynolds number $\operatorname{Re}_{\text {in }}=\rho Q /\left(\pi r_{\text {in }} \eta_{s}\right)<5 \times 10^{-2}$, the capillary number $\mathrm{Ca}_{\text {in }}=\eta_{s} Q /\left(\pi r_{\text {in }}^{2} \gamma\right)<10^{-3}$, and the Bond number $\mathrm{Bo}_{\text {in }}=\rho g r_{\text {in }}^{2} / \gamma=\mathcal{O}(1)$, with $g$ the gravitational acceleration. These estimates indicate that the suspensions detach only because of gravity. The capillary stress $\sigma_{\text {tip }}=$ $\gamma / r_{\text {in }} \simeq 39 \mathrm{~Pa} \gg \sigma_{c}$ stabilizes the drop forming at the tip of the needle. We waited $10 \mathrm{~min}$ after the suspensions had reached the needle tip to ensure steady flow and reproducibility. A high-speed camera (Phantom v7.3, Vision Research) either with a 105-mm macrolens and a bellows or mounted on a microscope recorded pinch-off events at frame rates between 5000 and 50000 frames per second.

Pinch-off is characterized by measuring the decrease with time of the minimal diameter $2 h_{\min }$ of the liquid bridge [17]. For Newtonian fluids, the capillary-driven dynamics are the result of an interplay between viscous and inertial stresses. The magnitude of the Reynolds number $\operatorname{Re}=\rho v_{t}(t) h_{\min }(t) / \eta_{s}$, with $v_{t} \propto \gamma / \eta_{s}$ the radial thinning speed, indicates the dominant terms in the stress balance. As Re depends on $h_{\min }$, the dynamics change during pinch-off [17]. Here, Re depends on the particle concentration $[C S]$, which influences $\eta_{s}$.

For $[C S]<29$ wt \%, we expect $\operatorname{Re} \gg 1$, and the measured thinning dynamics are in good agreement with the prediction $h_{\min } \simeq 0.7(\gamma / \rho)^{1 / 3}\left(t_{0}-t\right)^{2 / 3}$ for inviscid pinch-off [17], with $t_{0}$ the time at breakup [Fig. 1(b)]. Also, the rear part of the interface folds inward just before breakup [image 1 in Fig. 1(c)], which is a feature of inviscid pinch-off known as overturning [17].

For $29 \mathrm{wt} \% \leq[C S] \leq 33 \mathrm{wt} \%$, the observed thinning dynamics are linear in time [Fig. 1(b); e.g., image 2 in Fig. 1(c)], which is surprising as the suspensions in this range of concentrations display a non-Newtonian rheological response. The order of magnitude of the viscosity leads to $\operatorname{Re}=\mathcal{O}(1)$. Using the theoretical prediction $h_{\min }=$ $0.0304\left(\gamma / \eta_{s}\right)\left(t_{0}-t\right)$ [17] and the measured thinning rate, we deduce an apparent shear viscosity $\eta_{s} \simeq$ $50 \mathrm{mPa}$ s for the $30 \mathrm{wt} \%$ suspension, which agrees well with the value measured with the rheometer for shear rates $10 \mathrm{~s}^{-1} \leq \dot{\gamma} \leq 1000 \mathrm{~s}^{-1}$.

For $[C S]>33 \mathrm{wt} \%$, the thinning dynamics slow down when $h_{\min } \leq h_{\min , c}$, with $h_{\min , c}$ increasing from 0.9 to 
$1.5 \mathrm{~mm}$ with increasing [CS] [Fig. 1(b)]. The dynamics are well fitted by $h_{\min } \propto \exp \left(\alpha\left(t_{0}-t\right)\right)$ with a rate $\alpha$ that decreases when $[C S]$ increases [inset in Fig. 1(b)]. In addition, the bridge becomes cylindrical. Both these observations are signatures of viscoelasticity [4-6]. Close to breakup, fluctuations arise on the interface of the bridge [image 3a in Fig. 1(c)], which then thins in many locations with nonexponential dynamics, which we describe later. At breakup, the bridge splits into several threads that retract and buckle [image $3 \mathrm{~b}$ in Fig. 1(c)].

For viscoelastic pinch-off, the decay rate $\alpha$ is related to the longest relaxation rate of the liquid $\omega=3 \alpha$ [17]. Here, $\omega$ decreases from approximately 250 to $50 \mathrm{~s}^{-1}$ with increasing $[C S]$ [Fig. 2(a)]. In addition, the slowing down of the thinning dynamics is usually associated with an increase of the first normal stress difference $\tau_{z z}-\tau_{r r}$ in the bridge [18], with $\tau_{r r}$ and $\tau_{z z}$, respectively, the normal stresses along the radius and the axis of the bridge, respectively. In shear, at a gap width $w=h_{\min , c}$, we measured a significant positive first normal stress difference
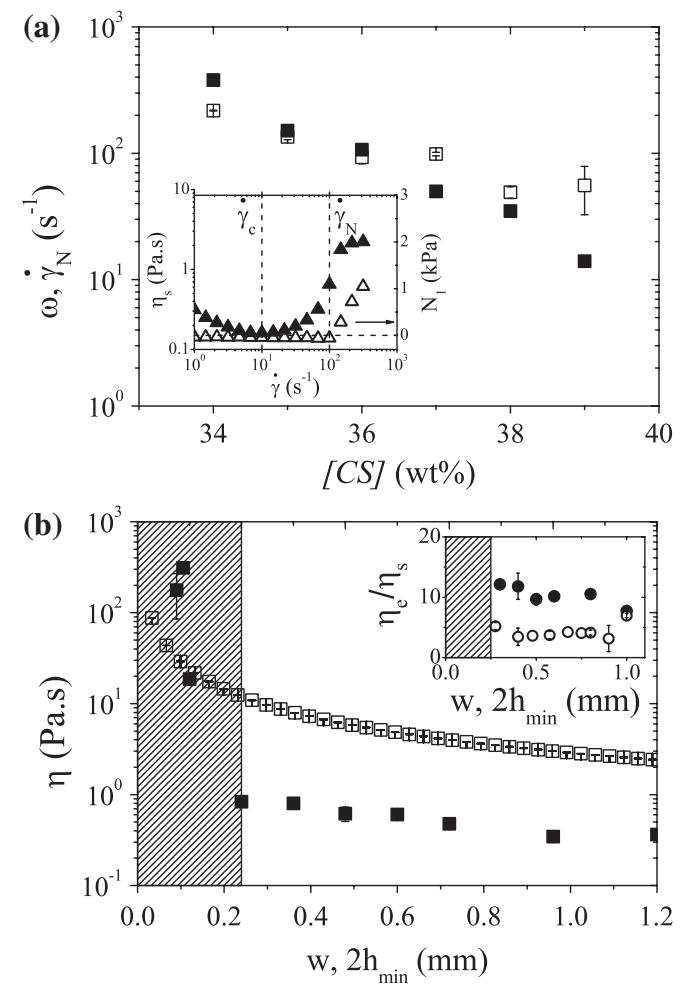

FIG. 2. (a) Comparison between the relaxation rate $\omega$ ( $\square$ ) obtained from the thinning dynamics and the shear rate $\dot{\gamma}_{N}$ (ם) as a function of $[C S]$. The gap width is $w=h_{\min , c}$. Inset: Apparent shear viscosity $\eta_{s}(\mathbf{\Delta})$ and first normal stress difference $N_{1}(\triangle)$ as a function of $\dot{\gamma}$ for $[C S]=36 \mathrm{wt} \%$ [see Fig. (b) in Supplemental Material [16]]. (b) Comparison between the extensional viscosity $\eta_{e}(\square)$ and the shear viscosity $\eta_{s}(\square)$ as a function of confinement $\left(2 h_{\min }\right.$ and gap width $\left.w\right)$ for $[C S]=$ 36 wt $\%$. Inset: Evolution of $\eta_{e} / \eta_{s}$ as a function of the confinement for $[C S]=36 \mathrm{wt} \%(\bigcirc)$ and $38 \mathrm{wt} \%(\bigcirc)$. In both graphs, the shaded area corresponds to the transition leading to breakup.
$N_{1}=\tau_{x x}-\tau_{y y}$ for shear rates $\dot{\gamma} \geq \dot{\gamma}_{N}$, with $\tau_{x x}$ and $\tau_{y y}$ the normal stresses along the velocity and the velocity gradient, respectively. Using the 50-mm plate tool, $N_{1}$ is measured with an accuracy of $1 \mathrm{~Pa}$, which is much smaller than the typical normal stress difference estimated in our experiments. The shear rate $\dot{\gamma}_{N}$ compares very well with $\omega$ for $34 \mathrm{wt} \%<[C S]<38 \mathrm{wt} \%$ [Fig. 2(a)] and is an order of magnitude greater than the shear rate at the onset of shear thickening $\dot{\gamma}_{c}$ [inset in Fig. 2(a)]. The good agreement between $\omega$ and $\dot{\gamma}_{N}$ reflects the importance of positive first normal stress differences in setting the time scale of the viscoelastic dynamics. The results also suggest that normal stresses are related to a similar, if not the same, microscopic mechanism in shear and in extension. Finally, suspensions in the range of concentration $29 \mathrm{wt} \%<$ $[C S]<32 \mathrm{wt} \%$ show viscous rather than viscoelastic pinch-off because the normal stresses become positive at time scales on the order of $1000 \mathrm{~s}^{-1}$, which can be reached here only very close to breakup.

Since confinement plays a role in the shear rheology of suspensions [10], we next identify these features for extensional flows through the relationship of $\eta_{e}$ and $\eta_{s}$. In particular, we compare extensional flows and shear flows with characteristic time scales equal to $\omega$. Both the extensional viscosity of the suspensions $\eta_{e}=\left(\tau_{z z}-\tau_{r r}\right) / \dot{\epsilon}$, which can be estimated by $\eta_{e}=\gamma /\left(h_{\min } \dot{\epsilon}\right)$ [19], and the shear viscosity $\eta_{s}$ measured at a constant shear rate $\dot{\gamma}=\omega$ increase when the suspensions are more and more confined [Fig. 2(b)]. In fact, the ratio $\eta_{e} / \eta_{s}$ is almost constant until both viscosities start to increase rapidly at the smallest confinements [inset in Fig. 2(b)]. For the highest concentrations, $\eta_{e} / \eta_{s} \simeq 3$, as found for Newtonian fluids. These observations suggest that, in addition to the choice of the correct time scale, confinement is important to be able to draw connections between shear and extensional flows of suspensions.

As indicated above, the bridge is cylindrical during the exponential regime, and its interface fluctuates as pinch-off is approached [image 3a in Fig. 1(c)]. A fluid cylinder can destabilize because of the Rayleigh-Plateau capillary instability [17]. Here, the fluctuations arise when $h_{0} \simeq$ $150 \mu \mathrm{m}$. An estimate of the thinning velocity is $v_{t}=$ $\alpha h_{0} \simeq 5 \mathrm{~mm} \mathrm{~s}^{-1}$. Because of confinement, $\eta_{s}$ has increased to $10 \mathrm{~Pa}$ s [Fig. 2(b)]. Under these conditions, we expect $\operatorname{Re} \ll 1$, and the most unstable mode of this instability, $\lambda_{m}=2 \pi h_{0} \sqrt{2+3 \sqrt{2 \eta^{2} /\left(\rho h_{0} \gamma\right)}} \simeq 7 \mathrm{~mm}$ [17], is much greater than the measured average wavelength $\lambda_{f} \simeq 700 \mu \mathrm{m}$, which varies little with $[C S]$. Therefore, the destabilization of the interface relies on a mechanism different from the capillary instability.

We uncovered this new mechanism by using a microscope tilted on its side coupled with a high-speed camera. The resolution at the highest magnification $(1.1 \mu \mathrm{m} / \mathrm{pixel})$ allowed us to follow individual gains during pinch-off [Fig. 3(a)] in threads as thick as $300 \mu \mathrm{m}$ even though the 

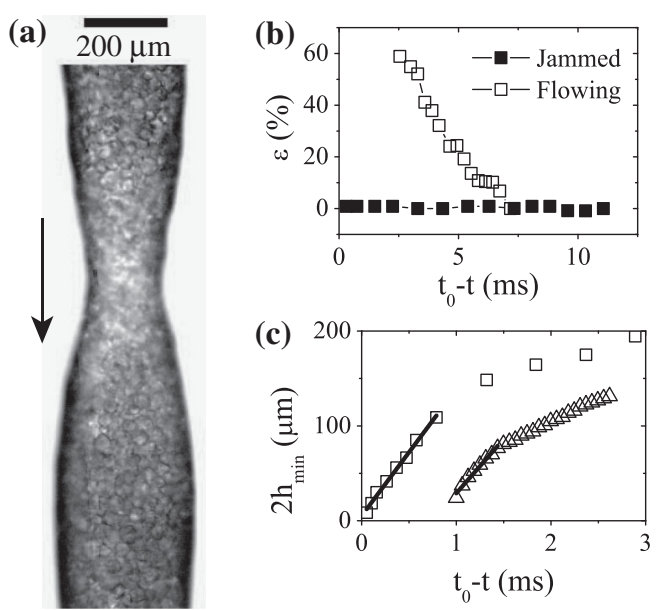

FIG. 3. (a) Image of the starch grains in the bridge close to breakup. (b) Strain $\epsilon$ versus $\left(t_{0}-t\right)$ for $[C S]=37 \mathrm{wt} \%$ in a jammed volume (ם) and in a flowing volume ( $\square$ ). (c) Thinning dynamics at breakup location for $[C S]=37 \mathrm{wt} \%(\triangle)$ and 39 wt $\%(\square)$. Continuous lines: linear fits to the data. The slope is $v_{t}=114 \mathrm{~mm} \mathrm{~s}^{-1}$ for $[C S]=37 \mathrm{wt} \%$ and $v_{t}=133 \mathrm{~mm} \mathrm{~s}^{-1}$ for $[C S]=39 \mathrm{wt} \%$. Time is shifted by $1 \mathrm{~ms}$ for $[C S]=$ $37 \mathrm{wt} \%$.

suspensions are opaque in bulk. The strain $\epsilon(t)=\frac{\ell(t)}{\ell_{0}}-1$ of pairs of grains aligned along the axis of the bridge, with $\ell_{0}$ the distance between two grains obtained from the first frame where they can be seen, remains almost constant and close to 0 in some "jammed" regions as the bridge thins, whereas it increases in other "flowing" regions [Fig. 3(b)]. The flow is heterogeneous.

As a consequence of this heterogeneity, for $h_{\min } \leq$ $100 \mu \mathrm{m}$, the thinning of the bridge proceeds only in the fluid volumes with a linear trend in time [Fig. 3(c)], eventually leading to breakup. Because of the viscosity increase due to confinement, we expect $\operatorname{Re} \ll 1$, and we fit these linear thinning dynamics with $h_{\min }=$ $0.0708\left(\gamma / \eta_{s, f}\right)\left(t_{0}-t\right)$ [17], with $\eta_{s, f}$ the apparent shear viscosity of the suspension during this regime. For both $[C S]=37 \mathrm{wt} \%$ and $39 \mathrm{wt} \%, v_{t} \simeq 100 \mathrm{~mm} \mathrm{~s}^{-1}$ and we obtain $\eta_{s, f} \simeq 85 \times 10^{-3} \mathrm{~Pa}$ s, which is much lower than the apparent shear viscosity $10 \mathrm{Pas}<\eta_{100}<1000 \mathrm{Pas}$ measured for the same suspensions with the plate-plate geometry at a gap width $w=100 \mu \mathrm{m}$ [Fig. 2(b)]. The discrepancy between $\eta_{s, f}$ and $\eta_{100}$ indicates that the suspensions experience self-dilution [14], i.e., the particle concentration decreases because of a significant difference between the flow of the solvent and that of the jammed particles. We note that self-dilution appears to occur locally and leads to nearly periodic surface fluctuations. From the rheological measurements, we estimate that the decrease in particle concentration is between 5 and $10 \mathrm{wt} \%$, which is the same order as the values reported for Brownian suspensions [14]. A change in the volume fraction has also been suggested to explain the acceleration (a)

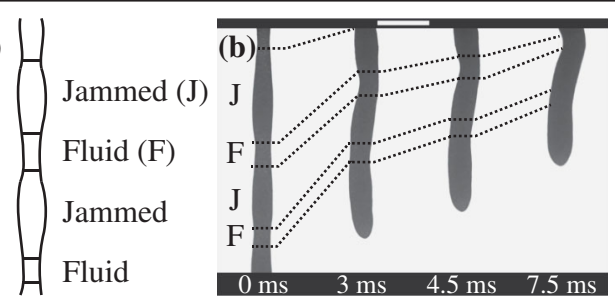

FIG. 4. (a) Sketch of the state of the suspension along the bridge close to breakup. (b) Images of a thread after breakup showing the bending of the thread around its fluid parts, $[C S]=37 \mathrm{wt} \%$ and $t_{r}=\omega^{-1} \simeq 10 \mathrm{~ms}$. Scale bar: $500 \mu \mathrm{m}$.

at the end of the thinning dynamics of non-shearthickening suspensions [20]. In our case, the viscosity during the last regime is very different from the viscosity of the $\mathrm{CsCl}$ solution, which means that we are measuring a property of the suspension.

The heterogeneous structure of the bridge just before breakup [Fig. 4(a)] allows us to explain the buckling of the threads formed after rupture. The threads retract on times shorter than the relaxation time $t_{r}$ of the jammed parts, $t_{r} \geq \omega^{-1} \simeq 10 \mathrm{~ms}$. To accommodate this time scale, they buckle around their deformable fluid volumes as observed in the experiments [Fig. 4(b)], where a thread bends around the regions that had the smaller diameters before breakup. We note that a bridge of a concentrated Brownian suspension can recoil in a fashion similar to the threads studied here [6]. Self-dilution was suggested as the supporting mechanism in this case.

In conclusion, we have studied the detachment of drops of concentrated suspensions of cornstarch that show continuous shear thickening. We show that the suspensions exhibit viscoelastic pinch-off dynamics provided the extension rate is sufficiently large to trigger normal stresses. Our results imply that normal stresses arise for the same microscopic reasons in shear and extension. By using particle tracking, we provide direct evidence that the flow of the suspensions in the bridge is heterogeneous, which suggests local variations in the particle volume fraction, consistent with self-dilution. This nonuniform structure is the cause of the buckling of the threads observed after breakup. Why the locations where self-dilution occurs are arranged in a nearly periodic fashion with a welldefined average size remains an open question.

The authors acknowledge M. Abkarian, L. Courbin, and P. Panizza for fruitful discussions. This work was funded by a grant from Unilever Research.

*hastone@princeton.edu

[1] V. Tirtaatmadja and T. Sridhar, J. Rheol. 37, 1081 (1993).

[2] H. A. Barnes, J. Rheol. 33, 329 (1989).

[3] A. A. Catherall, J. R. Melrose, and R. C. Ball, J. Rheol. 44, 1 (2000). 
[4] E. E. Bischoff White, M. Chellamuthu, and J. P. Rothstein, Rheol. Acta 49, 119 (2009).

[5] M. Chellamuthu, E. M. Arndt, and J.P. Rothstein, Soft Matter 5, 2117 (2009).

[6] M. I. Smith et al., Nature Commun. 1, 114 (2010).

[7] R. A. Bagnold, Proc. R. Soc. A 225, 49 (1954).

[8] J. Bergenholtz, J. F. Brady, and M. Vicic, J. Fluid Mech. 456, 239 (2002).

[9] J. R. Melrose and R. C. Ball, J. Rheol. 48, 937 (2004).

[10] A. Fall et al., Phys. Rev. Lett. 100, 018301 (2008).

[11] E. Brown and H. M. Jaeger, Phys. Rev. Lett. 103, 086001 (2009).

[12] E. Brown et al., Nature Mater. 9, 220 (2010).

[13] A. Fall et al., Phys. Rev. Lett. 105, 268303 (2010).

[14] M. D. Haw, Phys. Rev. Lett. 92, 185506 (2004).
[15] F. S. Merkt et al., Phys. Rev. Lett. 92, 184501 (2004).

[16] See Supplemental Material at http://link.aps.org/ supplemental/10.1103/PhysRevLett.107.134503 for (a) a plot of the shear stress $\sigma$ against the shear rate $\dot{\gamma}$, (b) an expanded version of the plot in the inset of Fig. 2(a) showing the first normal stress difference $N_{1}$ as a function of $\dot{\gamma}$.

[17] J. Eggers and E. Villermaux, Rep. Prog. Phys. 71, 036601 (2008).

[18] V.M. Entov and E. J. Hinch, J. Non-Newtonian Fluid Mech. 72, 31 (1997).

[19] A. N. Rozhkov, J. Eng. Phys. Thermophys. 45, 768 (1983).

[20] C. Bonnoit, E. Clément, and A. Lindner, arXiv: $1009.1819 \mathrm{v} 2$. 